\title{
Both Water Intoxication and Osmotic BBB Disruption Increase Brain Water Content in Rats
}

\author{
P. KOZLER ${ }^{1}$, V. RILJAK ${ }^{1}$, J. POKORNÝ ${ }^{1}$ \\ ${ }^{1}$ Institute of Physiology, First Faculty of Medicine, Charles University in Prague, Czech Republic
}

Received March 17, 2013

Accepted May 10, 2013

\section{Summary}

Our previous experiments revealed that water intoxication and osmotic BBB disruption in the rat allow penetration of highmolecular substances into the brain and that resulting changes in the internal environment of the CNS lead to pathological development, such as the loss of integrity of myelin. The aim of the present study was to determine whether the previously described phenomena are associated with increased water content in the brain. To answer the question following methods were used: a) water intoxication: intraperitoneal administration of distilled water, b) osmotic BBB disruption: application of mannitol $(20 \%)$ selectively into the internal carotid artery, c) brain wet weight was measured after decapitation, and subsequently (after six days in thermostat set at $86^{\circ} \mathrm{C}$ ) the dry weight were estimated d) in animals with $20 \%$ and $30 \%$ hyperhydration the degree of myelin deterioration was estimated e) animal locomotor activity was tested by continuous behavior tracking and analysis. Brain water content after water intoxication and following the administration of mannitol was higher than in the control group. Different degrees of hyperhydration led to different levels of brain water content and to different degrees of myelin impairment. Hyperhydration corresponding to $20 \%$ of the body weight brought about lower locomotor activity. Increased water content in the brain after the BBB osmotic disruption is surprising because this method is frequently used in the clinical practice.

\section{Key words}

Brain water content - Brain edema - Osmotic BBB disruption • Water intoxication $\bullet$ Locomotor activity

\section{Corresponding author}

Jaroslav Pokorný, Institute of Physiology, First Faculty of Medicine, Charles University in Prague, Albertov 5, 12800

Prague 2, Czech Republic. E-mail: pokorny@lf1.cuni.cz

\section{Introduction}

State of the internal environment of the nervous tissue is the result of instantaneous balance between diverse aspects of neuronal activity, activity of the glial elements, volume and properties of extracellular space and regulated permeability of the blood-brain barrier (BBB) (Pokorný et al. 2002). Our previous experiments revealed that properties of the internal environment of the brain can be changed by two experimental methods: water intoxication (hyperhydration) and osmotic disruption BBB. Such alteration of the internal environment of the brain allows high-molecular substances to cross the BBB, penetrate into the brain and induce neuropathological changes such as myelin disintegration (Kozler and Pokorný 2003, 2004, 2012, Kozler et al. 2010, 2011).

Water intoxication (hyperhydration) is used to induce cellular edema of the brain. It is a standard and currently used model (Olson et al. 1994, Vajda et al. 2000, Manley et al. 2000, Yamaguchi et al. 1997). Hyperhydration creates osmotic gradient and the water penetrates into the intracellular compartment, where it accumulates and increases the volume of the cell populations of the brain (Go 1997, Kimelberg 1995).

The idea of disruption the BBB by an osmotic insult was first accomplished by Rapoport (1970). By means of one-off intracarotid administration of a hyperosmolar solution shrinkage of endothelial cells is achieved, causing tight junctions to expand up to an average of 20 times their normal diameter. Owing to the distension of the tight junctions, BBB becomes permeable to high-molecular substances, which should be followed by water in the direction of osmotic gradient (Rapoport 2000). 
The aim of the present work was to find out whether the previously demonstrated changes in the internal environment of the brain induced by water intoxication or by osmotic $\mathrm{BBB}$ disruption are accompanied with increased water content in the brain.

\section{Material and Methods}

Adult Wistar strain laboratory rats of both sexes (weight 350-450 g) were used in our experiments. The maintenance and handling of experimental animals was in accordance with the EU Council Directive (86/609EEC) and the investigation was approved by the Expert Committee of the Institute of Physiology (of the First Medical Faculty, Charles University). For the estimation of water content, 30 animals were used (six animals in each group and six intact control animals). For the neurohistological study, experimental and control groups consisted of three animals each. In the behavioral part of the study, sixteen animals were used (two groups of 8 animals). Only adult male rats were used in this part of the experiment.

\section{Water intoxication}

For hyperhydration the standard model of water intoxication was used. Animals received distilled water in amount corresponding to $20 \%, 30 \%$ or $40 \%$ of the of their body weight. The volume was divided into three parts and administered intraperitoneally during 24 hours. The next day (16 hours after the last dose of distilled water), animals were taken into the experiments.

\section{Osmotic disruption of $B B B$}

For the microsurgical exposure of the internal carotid (ACI), each rat was put into the state of general anesthesia using intraperitoneal injection of thiopental ( $4 \mathrm{mg} / 100 \mathrm{~g}$ ) and allowed to ventilate spontaneously throughout the procedure. Starting from a skin incision along the midline between the upper end of the sternum and the mandible, the whole common carotid artery (ACC, arteria carotis communis) was exposed with a standard microsurgical technique and, beyond its bifurcation, also the proximal portions of the internal carotid (ACI, arteria carotis interna) and external carotid (ACE, arteria carotis externa), which was ligated close beyond the bifurcation. An intraluminal catheter was introduced into the ACC trunk from the arteriotomy for selective application of mannitol. With the application over and the catheter removed, the ACC was ligated distal to and proximal to the arteriotomy. The operation concluded with a single-layer suture (Kozler 2002).

Mannitol $20 \%$ (200 g in $1000 \mathrm{ml}$ of water for injection, $1098 \mathrm{mosmol} / \mathrm{l}$ ) in the dose of $5 \mathrm{ml} / \mathrm{kg}$ was selectively applied in the ACI at the rate of $0.12 \mathrm{ml} / \mathrm{s}$ (Rapoport 2000). After the surgical intervention, animals were placed back to their cages offering standard access to food and water.

\section{Estimation of the water content in the brain}

Animals were decapitated 8 hours after the last dose of distilled water or 30 minutes after mannitol administration. Brain was immediately removed, weighed (wet weight), placed in the thermostat at $86{ }^{\circ} \mathrm{C}$ for a period of six days, and then weighed again (dry weight). The water content in the brain was determined in percentiles using the equation: (wet weight - dry weight) / wet weight x 100 (Kamoun et al. 2009).

\section{Neurohistology}

Study of the myelin required perfusion fixation of the brain. Animals were sacrificed in a deep anesthesia via standard transcardial perfusion with a $4 \%$ solution of paraformaldehyde in $0.1 \mathrm{M}$ phosphate buffer $(\mathrm{pH}$ 7.4) for 15 minutes. After removal from the skull, the brain was fixed in the same solution for 24 hours. Serial coronary sections ( $30 \mu \mathrm{m}$ thick) were sliced by a vibratome from each brain, placed on gelatin-coated slides and dried.

The sections were rehydrated and axonal changes detected with the Black Gold II method of staining (Histo-Chem Inc., Jefferson, AZ, USA.) (Schmued and Slikker 1999). The hippocampal formation was the main part of the brain under study because of its known high sensitivity to various pathogenic stimuli. Analysis was centred on the CA1 and CA3 areas of the hippocampus and on the dorsal blade of the dentate gyrus (DG). The neurohistological picture of the axonal structural integrity was assessed with the aid of the following grades of myelin degradation: $1=$ no change, $2=$ sporadic edematous vesicles and sporadic edematous axons, $3=$ multiple vesicles, varicosity, edematous axons and helical course of axons, $4=$ myelin fragmentation. The degree of myelin damage was compared between the groups with hyperhydration corresponding to $20 \%, 30 \%$ and control animals.

\section{Behavior}

Only male Wistar albino rats were used in this part of the experiment. All experiments took place 
between 08:00 and 15:00 in a room with lights on (light intensity between 150 and $200 \mathrm{~lx}$ at the level of cages). During the tests, animals had not access to either food or water. Together three test session took place. Locomotor activity was observed firstly in intact animals (control session, baseline data); second session took place 48 hours after the beginning of hyperhydration (20\% of the body weight, given as three consecutive doses) and the last session took place seven days after hyperhydration. In each of mentioned session animals were placed and tested in Laboras apparatus (Metris B.V., Netherlands) to analyze their behavioral pattern for one hour. Laboras ${ }^{\mathrm{TM}}$ is automated system for continuous behavior tracking and analysis. Mechanical vibrations generated by animal (locomotion, rearing etc.) are transformed into electrical signal. Such signals are finally processed, classified and compared by with the predetermined characteristic patterns by Laboras software. During the measurement, animals were left undisturbed. After each of the test session animals were returned to their cages (food and water ad libitum).

\section{Statistics}

All data were statistically processed using nonparametric Kruskal-Wallis test for five independent samples. Pairs of samples (control and one of the experimental) were tested using nonparametric MannWhitney test for two independent samples. The results were compared between the control group (6) and the experimental groups (each 6 animals).

Data from Laboras software (three one hour sessions) were analyzed over ten minute intervals (0-10 min, 10-20 min, 20-30 min, 30-40 min, 40-50 min, $50-60 \mathrm{~min})$ to gain the detail information of animal's behavior. Each measured behavioral parameter was analyzed separately. Laboras data were subjected to nonparametric tests (because of non-Gaussian data distribution). To compare the differences between particular groups within 10 minutes intervals MannWhitney test was used, if $\mathrm{p}<0.05$ results were considered as significant.

\section{Results}

In the brains of hyperhydrated animals (HH 20\%) and in that with osmotic BBB disruption by mannitol (Man), water content was higher than in the control group (CG). Significant difference was observed between $\mathrm{CG}$ and $\mathrm{HH} 20 \%$ groups $(\mathrm{p}<0.01)$, and between
$\mathrm{CG}$ and a Man groups $(\mathrm{p}<0.01)$. No difference was found between the $\mathrm{HH}$ and Man groups (see Fig. 1).

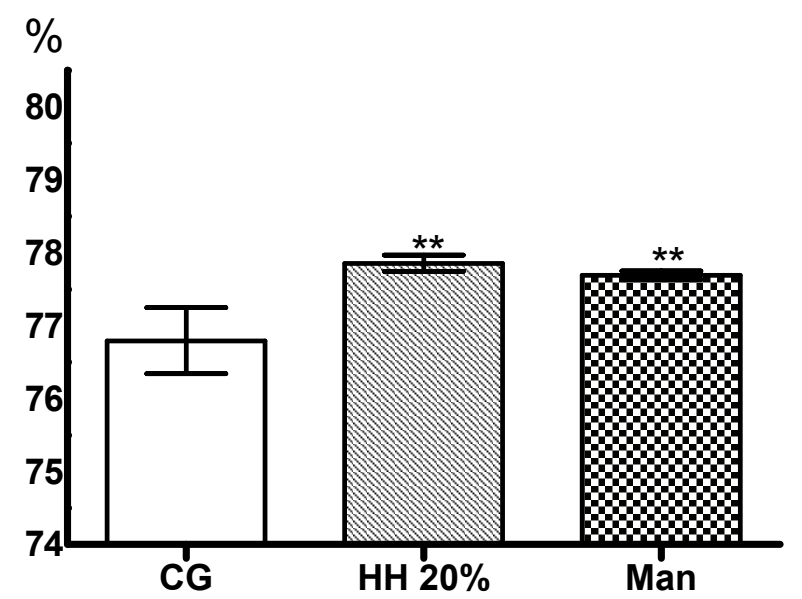

Fig. 1. Water content in the brain of hyperhydrated (HH 20\%), mannitol treated (Man) and control animals (CG). Significance of differences between experimental and control animals $(p<0.01)$ is given $\left({ }^{* *}\right)$.

Different levels of hyperhydration manifested by different water content in the brain. The larger was the volume of distilled water applied (HH 20\%, HH 30\% and $\mathrm{HH} 40 \%$ ) the higher water content in the brain was achieved. Difference between the control group (CG) and the hydrated groups $(\mathrm{p}<0.01)$ and among all hydrated groups (HH 20\% - 30\%, HH 30\% - 40\%, HH 20\%$40 \%$ ) were significant $(\mathrm{p}<0.01)$ (see Fig. 2).

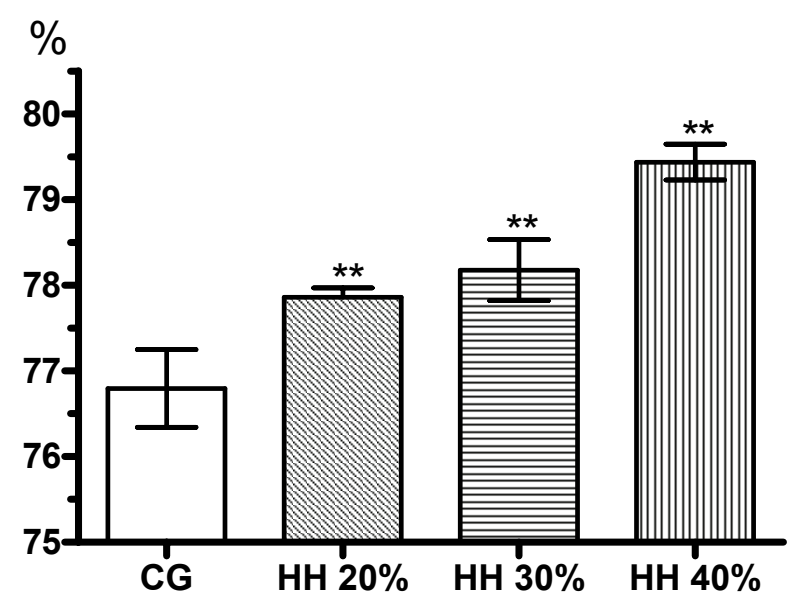

Fig. 2. Level of water content in the brain of the control (CG) and hyperhydrated animals ( $\mathrm{HH} 20 \%, \mathrm{HH} 30 \%$ and $\mathrm{HH} 40 \%$ ). Significance of differences between experimental and control animals $(p<0.01)$ is given $(* *)$.

Degree of myelin impairment reflected the level of hydration. The greater was the volume of distilled water that animals received, the more was the myelin 
disintegrated. Significant was the difference between the control group $(\mathrm{CG})$ and the hydrated groups $(\mathrm{p}<0.001)$ and among all hydrated groups (HH 20\%-30\%, HH $30 \%-40 \%$, HH 20\%-40\%) (p<0.001) (see Fig. 3).

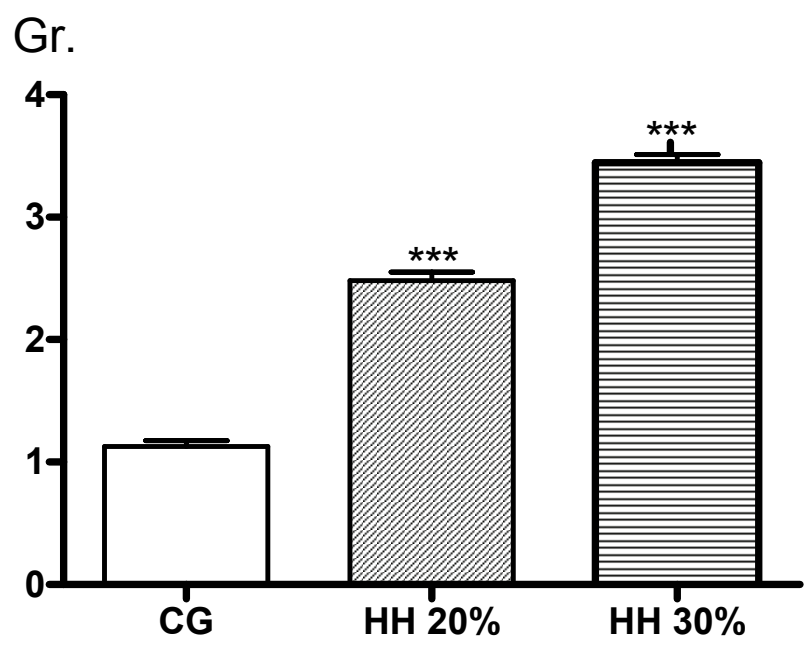

Fig. 3. Degree of myelin degradation in hyperhydrated animals ( $\mathrm{HH} \mathrm{20 \% ,} \mathrm{HH} 30 \%$ and $\mathrm{HH} \mathrm{40 \% )} \mathrm{compared} \mathrm{with} \mathrm{the} \mathrm{control}$ group (CG). Significance of differences between experimental and control animals $(p<0.001)$ is given $(* * *)$.

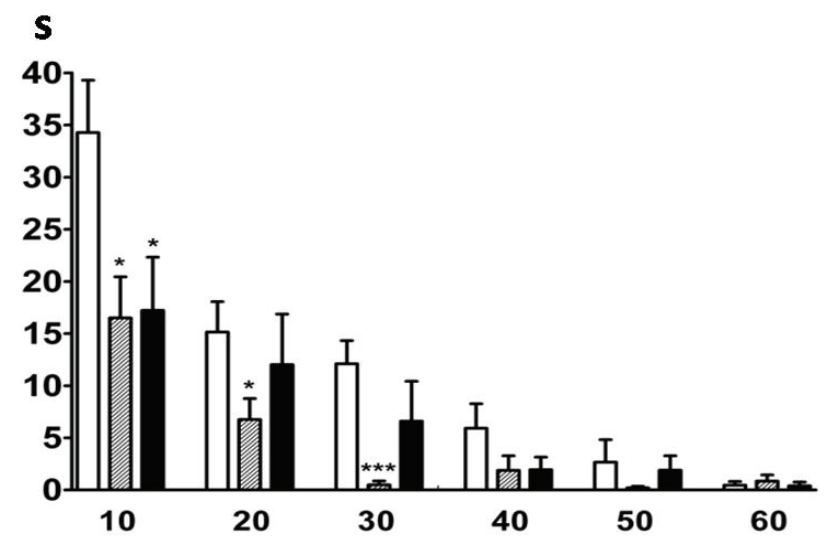

Fig. 4. Effect of hyperhydration ( $20 \%$ of the body weight) on the duration of locomotor activity (in seconds - s). Session lasting one hour is divided into six 10-minutes intervals. White columns represent control data (before hyperhydration), grey columns data represent animals after the hyperhydration, and black columns represent animals seven days after hyperhydration. Significance of differences to control animals at $\mathrm{p}<0.05$ is given $\left({ }^{*}\right)$; significance at $\mathrm{p}<0.001$ is given $(* * *)$. Error bars were calculated as \pm SEM.

Compared to naïve animals (measurement before hyperhydration, baseline), locomotor activity of the hyperhydrated animals was significantly lower in the first analyzed session. Animals after hyperhydration exhibited lower locomotor activity during initial part of the measurement (first $30 \mathrm{~min}$ ). Interestingly, one week after hyperhydration their activity returned back to nearly normal level. Figure 4 shows clearly, that locomotor activity was lowered in first half of test session (mutual comparison of the first and second test session, baseline vs. data obtained from the hyperhydrated animals). Duration of locomotion was significantly lower in hyperhydrated animals in the $1^{\text {st }}(\mathrm{p}<0.05), 2^{\text {nd }}(\mathrm{p}<0.05)$ and $3^{\text {rd }}(\mathrm{p}<0.001)$ test interval. Data reflect the fact, that exploratory activity was significantly lower immediately after hyperhydration, while habituation process was not affected by the treatment. Week after the treatment there was no significant difference between baseline data and hyperhydration effect, except immediately after the exposition to the new environment (first 10 minutes).

\section{Discussion}

Klatzo (1967) defined the cerebral edema as a condition with abnormal accumulation of water in the brain with its volume increasing. Kimelberg (1995) linked brain edema to the failure of homeostasis in the internal environment of the brain. Internal environment homeostasis of the brain is a basic condition for the normal function of CNS (Pokorny et al. 2002). Water intoxication and osmotic BBB disruption impair homeostasis of the internal environment of the brain and induce changes that can affect the function of the CNS. We demonstrated in experimental models using the above methods, penetration of high-molecular substances, namely Evans blue through the $\mathrm{BBB}$ and we have described its distribution in the extracellular and intracellular compartment (Kozler and Pokorný 2003), we have registered increased throughput via cytoplasmic membranes (Kozler and Pokorný 2004), and the loss of integrity of the myelin (Kozler et al. 2010).

We assumed that the described phenomena are initialized by the brain edema induced by osmotic disruption of BBB and/or by water intoxication. Hyperhydration (water intoxication), induced by intraperitoneally injected distilled water, is used as a standard experimental model of cellular brain edema. Edema can be confirmed by hyponatremia (Manley et al. 2000, Vajda et al. 2000, Yagamuchi et al. 1997). In our experiments, hyperhydration resulted in plasma levels of sodium lower than in healthy rats (by $20 \mathrm{mmol} / \mathrm{l})$ (Kozler and Pokorný 2003). It is therefore possible to conclude that water intoxication brings about higher water content in the brain with the real increase in brain volume - 
cellular edema. Not all authors studying the relationship between osmotic disruption of $\mathrm{BBB}$ and rise of water content in the brain came to the same conclusion. Rapoport et al. (1980) described transient increase of water content (1.0-1.5\%) after the osmotic disruption the BBB; Kaya et al. (2004) found significantly higher water content in rats with osmotically opened BBB. By contrast, Marchi et al. (2009) stressed that the osmotic disruption the BBB was followed by protein extravasation and the total water content of the brain was not increased. However, they admitted, that as a result of the accumulation of osmotically active substances, including proteins, in the extracellular space some exchange of water between the cellular and extracellularcompartment could develop. Similar findings published also Chi et al. (2000). It must be underlined that all the papers mentioned dealt with experimental models of osmotic disruption of BBB based on the selective administration of Mannitol into the internal carotid artery, the method that we also used. Our results showed that after the osmotic disruption the BBB by mannitol the water content significantly increased $(p<0.01)$ (see Fig. 1).

We can therefore speak about increase in brain volume and, according to Klatzo (1967) about the brain edema. In agreement with the new concept of brain edema (Kimelberg 1995) we believe, that in addition to proteins, the extracellular compartment is flooded with other solutes that eventually increase the brain extracellular osmolality and the resulting osmotic gradient between the blood and the tissue leads to the increase the brain water content. Method for osmotic disruption of BBB is clinical used for administration of high-molecular, water soluble drugs, namely cytostatics into the brain tissue. Osmotic disruption of BBB by mannitol and application of specific cytostatics via the opened BBB in patients in general anesthesia has been performed for many years (Gumerlock et al. 1992, Doolittle et al. 1998). Only as speculative, but likely reason for the use of the general anesthesia before the BBB disruption by Mannitol may be the concern of a sharp increase of water content in the brain with resulting edema.

Our findings of the higher water content in the brain in animals receiving large volumes of distilled water (see Fig. 2) can be explained by higher osmotic gradients after the hyperhydration. It helps to explain our results in the myelin study: The higher was hydration, the more severe the myelin integrity changes were - the degree of disintegrity myelin was closely related to the size of the edema (see Fig. 3). This result is consistent with the conclusion that axonal lesion are always preceded by the cellular edema (Onaya 2002).

Study of the behavior of experimental animals can be considered as a highly objective physical examination of the degree of brain impairment induced by edema (see Fig. 4). Results revealed evident reduction of locomotor activity in hyperhydrated animals. It correlates with the period of the highest water content in the brain (see Figs 1 and 4) and also with the period of the highest myelin disintegration (see Figs 3 and 4). Restoration of the locomotor activity after one week indicates that the initial stadium of brain edema which precedes axonal impairment is reversible (Gennarelli and Graham 1998, Sahuquillo et al. 2001).

\section{Conflict of Interest}

There is no conflict of interest.

\section{Acknowledgements}

Work was supported with grant P-34/LF1/7.

\section{References}

CHI OZ, LEE DI, LIU X, WEISS HR: The effects of morphine on blood-brain barrier disruption caused by intracarotid injection of hyperosmolar mannitol in rats. Anesth Analg 90: 603-608, 2000.

DOOLITTLE ND, PETRILlO A, BELL S, CUMMINGS P, ERIKSEN S: Blood-brain barrier disruption for the treatment of malignant brain tumors: The National Program. J Neurosci Nurs 30: 81-90, 1998.

GENNARELLI TA, GRAHAM DI: Neuropathology of the head injuries. Semin Clin Neuropsychiatry 3: 160-175, 1998.

GO KG: The normal and pathological physiology of brain water. Adv Tech Stand Neurosurg 23: 47-142, 1997.

GUMERLOCK MK, BELSHE BD, MADSEN R, WATTS C: Osmotic blood-brain barrier disruption and chemotherapy in the treatment of high grade malignant glioma: patient series and literature review. J Neurooncol 12: 33-46, 1992. 
KAMOUN WS, LEY CD, FARRAR CT, DUYVERMAN AM, LAHDENRANTA J, LACORRE DA, BATCHELOR TT, DI TOMASO E, DUDA DG, MUNN LL, FUKUMURA D, SORENSEN AG, JAIN RK: Edema control by cediranib, a vascular endothelial growth factor receptor-targeted kinase inhibitor, prolongs survival despite persistent brain tumor growth in mice. J Clin Oncol 20: 2542-2552, 2009.

KAYA M, GULTURK S, ELMAS I, KALAYCI R, ARICAN N, KOCYILDIZ ZC, KUCUK M, YORULMAZ H, SIVAS A: The effects of magnesium sulfate on blood-brain barrier disruption caused by intracarotid injection of hyperosmolar mannitol in rats. Life Sci 26: 201-212, 2004.

KIMELBERG HK: Current concepts of brain edema. Review of laboratory investigations. J Neurosurg 83: 1051-1059, 1995.

KLATZO I: Presidental address. Neuropathological aspects of brain edema. J Neuropathol Exp Neurol 26: 1-14, 1967.

KOZLER P: Osmotic disruption of the blood-brain barrier in an experiment. (in Czech) Rozhl Chir 80: 393-396, 2001.

KOZLER P, POKORNÝ J: Altered blood-brain barrier permeability and its effect on the distribution of Evans blue and sodium fluorescein in the rat brain applied by intracarotid injection. Physiol Res 52: 607-614, 2003.

KOZLER P, POKORNÝ J: Effects of intracarotid injection of methylprednisolone on cellular oedema after osmotic disruption of the blood-brain barrier in rats. Prague Med Rep 105: 279-290, 2004.

KOZLER P, POKORNY J: Effect of methylprednisolone on the axonal impairment accompanying cellular brain oedema induced by water intoxication in rats. Neuro Endocrinol Lett 28: 782-786, 2012.

KOZLER P, RILJAK V, POKORNY J: Time-dependent axonal impairment in experimental model of brain oedema. Neuro Endocrinol Lett 31: 477-482, 2010.

KOZLER P, RILJAK V, POKORNÝ J: Methylprednisolone reduces axonal impairment in the experimental model of brain oedema. Neuro Endocrinol Lett 32: 831-835, 2011.

MANLEY GT, FUJIMURA M, MA T: Aquaporin-4 deletion in mice reduces brain edema after acute water intoxication and ischemic stroke. Nat Med 6: 159-163, 2000.

MARCHI N, BETTO G, FAZIO V, FAN Q, GHOSH C, MACHADO A, JANIGRO D: Blood-brain barrier damage and brain penetration of antiepileptic drugs: role of serum proteins and brain edema. Epilepsia 50: 664-677, 2009.

OLSON JE, EVERS JA, BANKS M: Brain osmolyte content and blood-brain barrier water permeability surface area product in osmotic edema. Acta Neurochir Suppl 60: 571-573, 1994.

ONAYA M: Neuropathological investigation of cerebral white matter lesions caused by closed head injury. Neuropatology 22: 243-251, 2002.

POKORNÝ J, KOZLER P, TROJAN S: Nervous tissue internal environment and blood-brain barrier and their part in the processes of reparative neuroplasticity. (in Czech) Čes Slov Neurol Neurochir 65/98: 307-312, 2002.

RAPOPORT SI: Effect of concentrated solutions on bloodbrain barrier. Am J Physiol 219: 270-274, 1970.

RAPOPORT SI: Osmotic disruption of the blood-brain barrier: principles, mechanism, and therapeutic applications. Cell Moll Neurobiol 20: 217-230, 2000.

RAPOPORT SI, FREDERICKS WR, OHNO K, PETTIGREW KD: Quantitative aspects of reversible osmotic disruption of the blood-brain barrier. Am J Physiol 238: 421-431, 1980.

SAHUQUILLO J, POCA MA, AMOROS S: Current aspects of pathophysiology and cell dysfunction after severe head injury. Curr Pharm Des 7: 1475-1503, 2001.

SCHMUED L, SLIKKER W: Black-Gold: a simple, high-resolution histochemical label for normal and pathological myelin in brain tissue sections. Brain Res 837: 289-297, 1999.

VAJDA Z, PROMENEUR D, DÓCZI T, SULYOK E, FRØKIAER J, OTTERSEN OP, NIELSEN S: Increased aquaporin-4 immunoreactivity in rat brain in response to systemic hyponatremia. Biochem Biophys Res Commun 270: 495-503, 2000.

YAMAGUCHI M, YAMADA T, KINOSHITA I, WU S, NAGASHIMA T, TAMAKI N: Impaired learning of active avoidance in water-intoxicated rats. Acta Neurochir Suppl 70: 152-154, 1997. 